\title{
Pour en lire plus : La communication environnementale : enjeux, acteurs et stratégies.
}

Ghada Touir

\author{
(2) OpenEdition \\ Journals \\ Édition électronique \\ URL : https://journals.openedition.org/ere/6248 \\ DOI : $10.4000 /$ ere.6248 \\ ISSN : 2561-2271 \\ Éditeur \\ Centr'ERE
}

Référence électronique

Ghada Touir, «Pour en lire plus : La communication environnementale : enjeux, acteurs et stratégies. », Éducation relative à l'environnement [En ligne], Volume 16 - 1| 2021, mis en ligne le 10 mars 2021, consulté le 28 mai 2021. URL : http://journals.openedition.org/ere/6248 ; DOI : https://doi.org/ 10.4000/ere.6248

Ce document a été généré automatiquement le 28 mai 2021. 
Pour en lire plus : La communication environnementale : enjeux, acteurs et stratégies.

Ghada Touir

RÉFÉRENCE

Kane, Oumar. (2016). La communication environnementale : enjeux, acteurs et stratégies.

Paris : Éditions L'Harmattan, 132 pages 


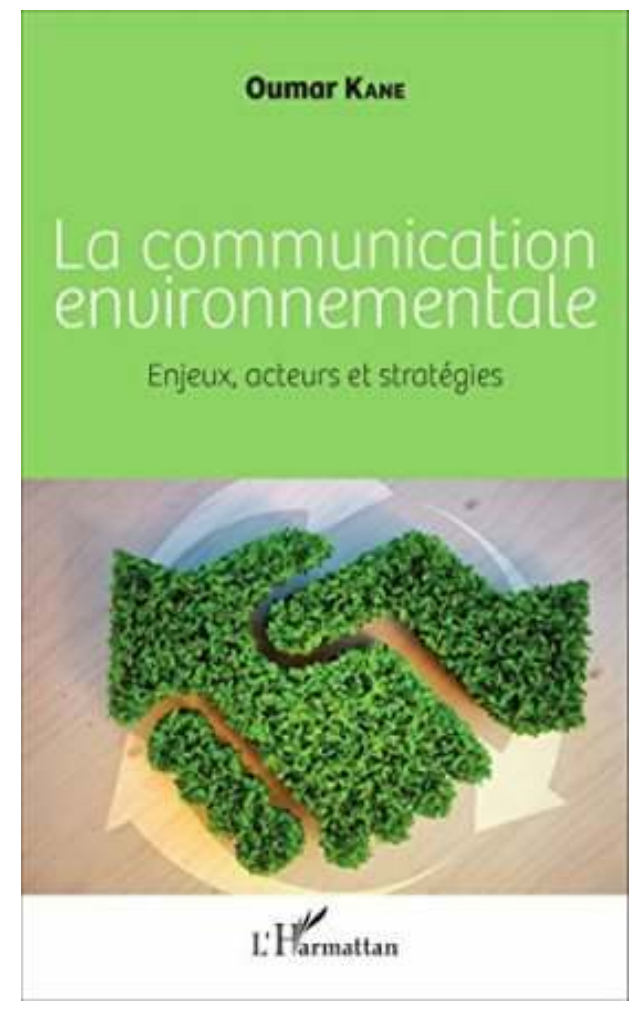

1 Il est difficile de dater avec précision l'émergence du champ de la communication environnementale. Certains spécialistes affirment, en lien avec le traitement médiatique de la catastrophe du naufrage du pétrolier Torrey Canyon le 18 mars 1967, que ce champ daterait d'environ une cinquantaine d'années. D'autres s'accordent pour situer son inscription dans le monde de la communication et de la sphère publique au milieu des années 1980, notamment à l'occasion de plusieurs crises comme la catastrophe de Bhopal en Inde en 1984, l'explosion du réacteur nucléaire de Tchernobyl en 1986, l'accident de l'usine chimique Sandoz de Bâle en Suisse en 1986 ou encore, lors de l'apparition de certaines problématiques environnementales majeures telles que la destruction de la couche d'ozone (Protocole de Montréal en 1987), la déforestation ou les changements climatiques, qui ont été traitées notamment dans des colloques et conférences internationales (comme celles des COP) et des sommets onusiens (comme celui de Rio de Janeiro en 1992).

2 La communication environnementale, comme champ de recherche interdisciplinaire, se développe, se structure et se formalise de plus en plus ces dernières années. Elle inclut selon L'International Environmental Communication Association (IECA, traduction libre) ${ }^{1}$, «toutes les diverses formes de la communication interpersonnelle, de groupe, publique, organisationnelle et médiatisée qui composent le débat social concernant les enjeux et les problèmes environnementaux, et notre relation avec le reste de la nature ».

3 C'est ainsi que plusieurs ouvrages contemporains portent sur la "communication environnementale », dont celui d'Oumar Kane paru en 2016 aux Éditions L'Harmattan et intitulé La communication environnementale : enjeux, acteurs, et stratégies ${ }^{2}$.

4 En 132 pages et 3 sections, qui peuvent se lire séparément, mais gagnent assurément à être consultées globalement, l'auteur s'appuie sur diverses références théoriques pour expliciter le rôle de la communication dans la gouvernance des questions liées à 
l'environnement. Il insiste d'abord sur la nécessité de repenser l'environnement comme un bien commun (plutôt que comme un bien public) et souligne, dans le contexte environnemental actuel, l'importance de l'approche interdisciplinaire en communication environnementale. Puis il explicite la notion de "public» afin de clarifier les modalités de mise en place des débats publics sur les questions environnementales à l'ère de la gouvernance institutionnelle existante. Il traite ensuite de la communication environnementale à travers une clarification des notions de développement durable, de communication de risque et de crise, à la fois du point de vue des institutions [avec la question du lobbying] et celui des organisations, en relation avec le marketing, l'écoblanchiment ou le greenwashing. L'auteur aborde également la question de l'expertise savante (neutre et politisée). Oumar Kane rappelle enfin le rôle important du public et des citoyens dans les débats sociaux en lien avec l'environnement.

5 Chaque partie de cet ouvrage fait référence à une vaste littérature sur le sujet et explicite, dans une perspective interdisciplinaire en lien avec l'environnement, différents concepts du domaine de la communication environnementale : la dichotomie bien public/bien commun, le changement environnemental, la gouvernance, les stratégies relevant d'approches descendantes (dite top down) et ascendantes (dite bottom up), l'éthique, les notions de risque et de crise, l'actualité médiatique (agendasetting), de même que les concepts de public, de pratiques, de participation, de contrat de communication, d'espace public, d'expertise et de consensus/dissensus.

6 Tout au long des chapitres, le professeur Kane peaufine la définition de la communication environnementale dans le monde contemporain. Il s'agit d'un champ complexe qui fait appel à l'interdisciplinarité, allant d'une perspective globale, soit « un champ d'études qui se situe à l'articulation des théories de la communication et des théories de l'environnement dans le but d'analyser le rôle et l'influence de la communication dans les problématiques environnementales » (p. 45), à la perspective spécifique de cette "crise qui est liée aux graves problèmes environnementaux auxquels l'humanité fait face» (p. 114). Ici, les médias jouent un rôle important, notamment dans l'éducation du public sur les enjeux environnementaux. L'auteur rappelle une distinction importante entre les médias traditionnels (comme la radio et la télévision) et les médias socionumériques (comme Facebook et Twitter) en ce qui a trait à l'influence auprès du public.

7 Dans sa démarche d'appréhension de la dimension interdisciplinaire de la communication environnementale, Oumar Kane utilise un exemple central, celui des changements climatiques: «l'une des préoccupations sociétales les plus importantes de la période actuelle»(p. 12). L'auteur s'appuie sur l'exemple du Groupe d'experts intergouvernemental sur l'évolution du climat (le GIEC ${ }^{3}$, cet organisme international créé en 1988 en vue de fournir des évaluations et des analyses scientifiques objectives et rigoureuses sur les changements climatiques), pour pointer les " climatosceptiques » qui « nient la réalité du changement climatique et qui invoquent parfois la théorie du complot» (p. 117).

8 Au bilan, cet ouvrage aborde avec pertinence une thématique bien d'actualité et offre des éclairages importants aux étudiant-e-s et aux chercheur-e-s sur la notion et plus généralement, sur le champ de la communication environnementale ${ }^{4}$. Il ouvre aussi toute grande la porte à des recherches contemporaines en communication environnementale dans le monde francophone. Au-delà de ces apports, signalons 
toutefois qu'il aurait été probablement intéressant d'inclure davantage d'études de cas (provenant de différents pays et contextes) interpellant la communication environnementale. Également, il serait possible d'ouvrir sur tout un champ interdisciplinaire complémentaire et qui a plus de cinquante ans, celui de l'éducation relative à l'environnement (ERE).

\section{NOTES}

1. Mark Meisner (2015). Environmental communication: What it is and why it matters - https:// theieca.org/resources/environmental-communication-what-it-and-why-it-matters

2. Ce livre est la synthèse d'une recherche subventionnée par le Fonds de recherche du Québec sur la société et la culture (FRQSC) portant sur les relations entre la communication et l'environnement

3. Fiche d'information sur le GIEC : Qu'est-ce que le GIEC ? (2013) - https://www.ipcc.ch/site/ assets/uploads/2018/04/FS_what_ipcc_fr-1.pdf

4. Notons au passage la parution au cours de la même année (2016), d'un ouvrage collectif consacré également la communication environnementale »: Thierry Libaert (2016) (Dir), La communication environnementale. Éditions du CNRS, Collection «Les Essentiels d'Hermès », 263 p. ISBN : 978-2-271- 09351-6.

\section{AUTEUR}

GHADA TOUIR

Université Concordia, Québec 